\title{
Transtorno da excitação genital persistente: uma revisão da literatura
}

\author{
Persistent genital arousal disorder: a literature review \\ Valeska Martinho Pereira', Adriana Cardoso de Oliveira e Silva ${ }^{2}$, Antonio Egidio Nardi
}

\author{
Palavras-chave \\ Sexualidade, disfunção \\ sexual fisiológica, \\ disfunções sexuais \\ psicogênicas, diagnóstico.
}

\section{Keywords}

Sexuality, physiological

sexual dysfunction,

psychogenic sexual

dysfunction, diagnosis.

\section{RESUMO}

Objetivo: Realizar uma revisão da literatura sobre o transtorno da excitação genital persistente (TEGP), um quadro clínico que acomete somente mulheres, recentemente descrito na literatura, caracterizado por sinais fisiológicos de excitação sexual sem a presença de desejos ou estímulos sexuais. Métodos: Foi realizada revisão sistemática com busca nas bases científicas PubMed, ISI, SciELO e PsycInfo. Do total de artigos encontrados, 27 foram selecionados para integrar esta revisão. Resultados: Os artigos, em sua maioria, são relatos de casos. Apesar de algumas hipóteses diagnósticas terem sido propostas, ainda não existe consenso sobre etiologia, fatores de risco e epidemiologia desse transtorno. A literatura aponta para uma correlação positiva entre a presença do transtorno e quadros depressivos, ansiosos e sintomas obsessivo-compulsivos. Conclusão: Há necessidade de estudos mais amplos para o melhor entendimento desse quadro clínico. É possível que muitos casos passem despercebidos pelos profissionais de saúde por desconhecimento dos critérios diagnósticos.

\begin{abstract}
Objective: To review the literature on persistent genital arousal disorder, a clinical syndrome that affects only women, recently described in the literature, characterized by symptoms of physiological arousal in the absence of desire or sexual stimulation. Methods: We performed a systematic review and search of the scientific basis PubMed, ISI, SciELO and PsycInfo. Of the total number of articles found, 27 were selected to incorporate this review. Results: Most articles are reports of cases. Although some diagnostic hypotheses have been proposed, there is no consensus yet on the etiology, risk factors, and epidemiology of the disorder. The literature suggests a positive relationship between the presence of the disorder and depression, anxiety and obsessive-compulsive symptoms. Conclusion: There is a need for further studies for better understanding of this clinical picture. It is possible that many cases go unnoticed by health professionals for lack of knowledge of diagnostic criteria.
\end{abstract}

1 Universidade Federal do Rio de Janeiro (UFRJ), Instituto de Psiquiatria (IPq), Programa de Pós-Graduação em Psiquiatria e Saúde Mental; Laboratório de Pânico e Respiração, UFRJ-IPq; INCT Translational Medicine.

2 UFRJ-IPq, Programa de Pós-Graduação em Psiquiatria e Saúde Mental e Laboratório de Pânico e Respiração; Universidade Federal Fluminense (UFF); INCT Translational Medicine.

3 UFRJ-IPq, Faculdade de Medicina, Programa de Pós-Graduação em Psiquiatria e Saúde Mental e Laboratório de Pânico e Respiração; INCT Translational Medicine.

Endereço para correspondência: Valeska Martinho Pereira. Universidade Federal do Rio de Janeiro, Instituto de Psiquiatria, Laboratório de Pânico e Respiração. Rua Visconde de Pirajá, 407/702 22410-003 - Rio de Janeiro, RJ Telefone: (21) 2521-6147/Telefax: (21) 2523-6839

E-mail: valeskapereirapsi@gmail.com 


\section{INTRODUÇÃO}

Disfunções sexuais são definidas pelo DSM-IV-TR' como "perturbação nos processos que caracterizam o ciclo de resposta sexual ou por dor associada com o intercurso sexual"; já a $\mathrm{CID}-10^{2}$ define que "as disfunções sexuais dizem respeito às diferentes manifestações segundo as quais um indivíduo é incapaz de participar numa relação sexual, como ele ou ela desejaria. A resposta sexual é um processo psicossomático e mais comumente processos tanto psicológicos quanto somáticos intervêm na causa da disfunção sexual". Os dois são os sistemas de classificação vigentes mais utilizados em pesquisas e na prática clínica.

O ciclo de resposta sexual definido por Masters e Johnson ${ }^{3}$, aperfeiçoado por Kaplan ${ }^{4}$ e que serve de base para os critérios diagnósticos das disfunções sexuais, divide-se em quatro fases: desejo, excitação, orgasmo e resolução. Cada fase apresenta características fisiológicas distintas e é possível que apenas uma das fases esteja comprometida, sem que as outras sejam prejudicadas em seu funcionamento.

A fase da excitação é descrita como "(...) um sentimento subjetivo de prazer sexual e alterações fisiológicas concomitantes"4. Nessa fase, há uma vasodilatação das arteríolas presentes nos órgãos sexuais, o que causa intumescência deles e os prepara para o coito. Na mulher, a vagina se distende e se lubrifica para acomodar o pênis.

O DSM-IV classifica como transtorno específico dessa fase o transtorno da excitação sexual feminina (TESF), caracterizado por incapacidade de manter ou obter uma resposta adequada de excitação sexual, com prejuízo da lubrificação e vasocongestão genital'.

As definições atuais dos transtornos sexuais têm foco nas respostas genitais. Em 2004, Basson et al..$^{5}$ publicaram um trabalho em que revisavam as definições das disfunções sexuais femininas 5 . Nesse artigo, define-se que a excitação sexual feminina pode ser classificada como subjetiva e genital e que existe uma falta de correlação entre a excitação genital e suas respostas fisiológicas e a excitação subjetiva. Mulheres podem apresentar respostas fisiológicas características da excitação e não se perceberem sexualmente excitadas.

A partir desse novo entendimento, os transtornos relacionados à fase da excitação foram divididos em quatro: transtorno da excitação sexual subjetiva; transtorno da excitação sexual genital e subjetiva combinada; transtorno da excitação sexual genital; e, mais recentemente, vemos surgir na literatura uma condição que afeta somente mulheres, o transtorno da excitação genital persistente.

O presente estudo objetiva fazer uma revisão da literatura existente. Verificar-se-á de que forma se configura o transtorno, suas características fisiológicas e psicológicas, diferenciando-o dos demais transtornos da excitação. Apresentar-se-á a configuração atual dos seus critérios diagnósticos, hipóteses etiológicas e possibilidades de tratamento.
É possível que muitos casos da doença tenham passado despercebidos por clínicos por causa do desconhecimento desses profissionais sobre o problema. Não existem dados de prevalência e é possível que a condição não seja tão rara quanto se pensou a princípio.

\section{Critérios diagnósticos}

Desde sua primeira descrição em 20016 , os critérios diagnósticos do transtorno da excitação genital persistente foram estabelecidos como:

1. Sinais característicos de excitação sexual (plenitude genital/inchaço e sensibilidade com ou sem ereção ou inchaço do mamilo) que persistem por um período extenso de tempo (horas ou dias) e não desaparecem completamente por si só.

2. Sinais de excitação fisiológica, os quais não se resolvem com experiências orgásticas habituais e podem exigir orgasmos múltiplos por horas ou dias para desaparecerem.

3. Sinais de excitação são geralmente experimentados e não estão relacionados a nenhum sentimento subjetivo de excitação ou desejo sexual.

4. A excitação genital persistente pode ser disparada não somente por atividade sexual, mas aparentemente também por estímulos não sexuais e até mesmo nenhum estímulo aparente.

5. Sinais são experimentados como espontâneos, intrusivos e indesejados e provocam na mulher sentimentos de angústia e preocupação.

Apesar da semelhança com o quadro de hipersexualidade, existe um fator diferenciador entre as duas condições. A hipersexualidade é caracterizada por um aumento excessivo do desejo sexual. Nas mulheres com transtorno da excitação genital persistente, não existe um relato de aumento do desejo sexual. A literatura ${ }^{7}$ indica que essas mulheres experimentam redução no desejo e interesse sexual, por receio de iniciar um episódio do transtorno.

\section{METODOLOGIA}

Foram realizadas buscas nas bases de dados PubMed, SciELO, PsycInfo e ISI Web of Knowledge utilizando os seguintes termos: "persistent", "sexual", "genital", "arousal", "disorder", "restless genital", "syndrome". Os termos usados na busca referem-se às diferentes propostas de nomenclatura que a condição recebeu desde seu primeiro relato na literatura ${ }^{6}$.

A pesquisa foi realizada em agosto de 2010, por dois pesquisadores independentes, sem restrição temporal em nenhuma base de dados. Os resultados das buscas foram comparados entre os dois pesquisadores. Foram excluídos trabalhos de revisão. 


\section{RESULTADOS}

No PubMed, utilizando os termos, foram encontrados 62 artigos. Após análise dos resumos dos artigos encontrados, 34 artigos foram recuperados e analisados. Dois artigos encontrados são revisões do tema e não foram incluídos neste estudo. Duas cartas aos autores foram incluídas por conter relatos de casos que não estavam presentes em outros artigos revistos. Seis artigos, apesar de citarem o transtorno, não o tinham como foco de estudo.

Na base ISI Web of Knowledge, foram recuperados 36 artigos. Após análise dos abstracts, somente 23 artigos foram aproveitados, e 4 das referências eram cartas ou comentários de artigos publicados e 1 era resumo de trabalho publicado em conferência científica.

Para minimizar possíveis perdas de artigos relevantes para a revisão, foi montada uma tabela com os resultados das buscas por dois pesquisadores, comparando-se os achados (Tabela 1). Verificaram-se manualmente os artigos identificados nas duas buscas e aqueles que foram identificados em apenas uma delas.

No total, foram encontradas 33 referências, sendo 2 artigos de revisão e 4 comentários ou cartas aos autores. Com isso, 27 artigos foram incluídos nesta revisão. Não foram identificados artigos em outras bases de dados.

Tabela 1. Estudos publicados sobre o Transtorno de Excitação Genital Persistente (TEGP)

\begin{tabular}{|c|c|c|c|c|c|}
\hline Data & Nome & Autores & Periódico & $\begin{array}{l}\text { Número de } \\
\text { participantes }\end{array}$ & Resumo \\
\hline 2001 & $\begin{array}{l}\text { Persistent sexual arousal syndrome: a newly } \\
\text { discovered pattern of female sexuality }\end{array}$ & Leiblum SR, Nathan SG & J Sex Marital Ther & 5 & $\begin{array}{l}\text { Por meio da descrição de cinco casos, os autores apresentam } \\
\text { um novo quadro clínico, caracterizado por uma excitação se- } \\
\text { xual que persistia por dias, apesar dos esforços para dissipá-la }\end{array}$ \\
\hline 2005 & $\begin{array}{l}\text { Persistent sexual arousal syndrome associa- } \\
\text { ted with increased soy intake }\end{array}$ & $\begin{array}{l}\text { Amsterdam A, Abu- } \\
\text { Rustum N, Carter J, } \\
\text { Krychman M }\end{array}$ & J Sex Med & 1 & $\begin{array}{l}\text { Descrição de um caso em que os sintomas iniciaram com um } \\
\text { aumento da ingestão de soja, com remissão mediante ajuste } \\
\text { da dieta }\end{array}$ \\
\hline 2005 & $\begin{array}{l}\text { Persistent sexual arousal syndrome: a } \\
\text { descriptive study }\end{array}$ & $\begin{array}{l}\text { Leiblum S, Brown C, Wan J, } \\
\text { Rawlinson L }\end{array}$ & J Sex Med & 103 & $\begin{array}{l}\text { Por meio de um questionário baseado na internet, os autores } \\
\text { buscaram identificar características do transtorno e possíveis } \\
\text { gatilhos }\end{array}$ \\
\hline 2006 & $\begin{array}{l}\text { Successful use of electroconvulsive therapy } \\
\text { in } 2 \text { cases of persistent sexual arousal } \\
\text { syndrome and bipolar disorder }\end{array}$ & $\begin{array}{l}\text { Yero SA, McKinney T, } \\
\text { Petrides G, Goldstein I, } \\
\text { Kellner CH }\end{array}$ & JECT & 2 & $\begin{array}{l}\text { Estudo de caso. Duas pacientes com diagnóstico de transtorno } \\
\text { bipolar e TGEP foram tratadas com eletroconvulsoterapia, } \\
\text { obtendo alívio em seus sintomas }\end{array}$ \\
\hline 2006 & $\begin{array}{l}\text { Sleep exacerbation of persistent sexual } \\
\text { arousal syndrome in a postmenopausal } \\
\text { woman }\end{array}$ & $\begin{array}{l}\text { Wylie K, Levin R, Hallam- } \\
\text { Jones R, Goddard A }\end{array}$ & J Sex Med & 1 & $\begin{array}{l}\text { Apresentação de caso em que o sujeito apresentava uma } \\
\text { piora dos sintomas ao dormir, descrevendo o caso clínico e } 0 \\
\text { tratamento }\end{array}$ \\
\hline 2006 & $\begin{array}{l}\text { Withdrawal of selective serotonin reuptake } \\
\text { inhibitors (SSRIs) may cause increased atrial } \\
\text { natriuretic peptide (ANP) and persistent } \\
\text { sexual arousal in women? - letter }\end{array}$ & $\begin{array}{l}\text { Goldmeier D, Bell C, } \\
\text { Richardson D }\end{array}$ & J Sex Med & 1 & $\begin{array}{l}\text { Discussão sobre o TEGP como possível efeito colateral da } \\
\text { retirada de medicação ISRS }\end{array}$ \\
\hline 2006 & $\begin{array}{l}\text { Persistent sexual arousal syndrome (PSAS): } \\
\text { possible etiologies and potential therapies }\end{array}$ & Craig HR & $\begin{array}{l}\text { Divulgação em } \\
\text { ambiente virtual }\end{array}$ & - & $\begin{array}{l}\text { Apresentação de possíveis causas etiológicas para o transtor- } \\
\text { no. Trabalho teórico }\end{array}$ \\
\hline 2007 & $\begin{array}{l}\text { Persistent sexual arousal in a woman with } \\
\text { associated cardiac defects and raised atrial } \\
\text { natriuretic peptide }\end{array}$ & $\begin{array}{l}\text { Bell C, Richardson D, } \\
\text { Goldmeier D, Crowley T, } \\
\text { Kocsis A, Hill S }\end{array}$ & Int J STD AIDS & 1 & $\begin{array}{l}\text { Estudo de caso com discussão sobre o papel do peptídeo } \\
\text { natriurético atrial no desenvolvimento e manutenção do TEGP }\end{array}$ \\
\hline 2007 & $\begin{array}{l}\text { Psychological, medical, and pharmacological } \\
\text { correlates of persistent genital arousal } \\
\text { disorder }\end{array}$ & $\begin{array}{l}\text { Leiblum S, Seehuus M, } \\
\text { Goldmeier D, Brown C }\end{array}$ & J Sex Med & 156 & $\begin{array}{l}\text { Por meio de um questionário baseado na internet, os autores } \\
\text { buscaram identificar correlatos psicológicos, médicos e } \\
\text { farmacológicos do TEGP }\end{array}$ \\
\hline 2007 & $\begin{array}{l}\text { Persistent genital arousal: disordered or } \\
\text { normative aspect of female sexual response? }\end{array}$ & $\begin{array}{l}\text { Leiblum S, Seehuus M, } \\
\text { Brown C }\end{array}$ & J Sex Med & 388 & $\begin{array}{l}\text { Por meio de um questionário baseado na internet, os autores } \\
\text { buscaram identificar mulheres que avaliam os sinais como } \\
\text { normais e se há diferenças entre mulheres que atendem } \\
\text { a todos os critérios do transtorno e aquelas que atendem } \\
\text { somente a alguns }\end{array}$ \\
\hline 2007 & $\begin{array}{l}\text { Normal and persistent genital arousal in } \\
\text { women: new perspectives }\end{array}$ & Leiblum SR, Chivers ML & J Sex Marital Ther & - & $\begin{array}{l}\text { Por meio da análise das respostas em um questionário on-line } \\
\text { publicado anteriormente, as autoras propõem que existam } \\
\text { três tipos de excitação genital na mulher e os fatores envolvi- } \\
\text { dos na avaliação da excitação }\end{array}$ \\
\hline 2007 & $\begin{array}{l}\text { Persistent sexual arousal syndrome: a case } \\
\text { report and review of the literature }\end{array}$ & Mahoney S, Zarate Jr C & J Sex Marital Ther & 1 & $\begin{array}{l}\text { Descrição de um caso de uma paciente bipolar que, após o uso } \\
\text { de venlafaxina, relatou sintomas parecidos aos do TEGP }\end{array}$ \\
\hline 2008 & $\begin{array}{l}\text { Interaction of organic and psychological } \\
\text { factors in persistent genital arousal disorder } \\
\text { in women: a report of six cases }\end{array}$ & Goldmeier D, Leiblum S & Int J STD AIDS & 6 & $\begin{array}{l}\text { Relato de uma série de seis casos de TEGP com discussão sobre } \\
\text { o papel da ansiedade na manutenção do transtorno }\end{array}$ \\
\hline 2008 & $\begin{array}{l}\text { Pelvic congestion syndrome presenting as } \\
\text { persistent genital arousal: a case report }\end{array}$ & Thorne C, Stuckey B & JSex Med & 1 & $\begin{array}{l}\text { Descrição de um caso em que a paciente apresentava altera- } \\
\text { ções nos vasos sanguíneos da pélvis }\end{array}$ \\
\hline 2008 & $\begin{array}{l}\text { Persistent genital arousal disorder in } \\
\text { women: case reports of association with } \\
\text { anti-depressant usage and withdrawal }\end{array}$ & Leiblum S, Goldmeier D & J Sex Marital Ther & 5 & $\begin{array}{l}\text { Por meio da descrição de cinco casos, os autores discutem } \\
\text { o papel das medicações antidepressivas no surgimento e } \\
\text { manutenção do transtorno }\end{array}$ \\
\hline
\end{tabular}


Tabela 1. Estudos publicados sobre o Transtorno de Excitação Genital Persistente (TEGP) (continuação)

\begin{tabular}{|c|c|c|c|c|c|}
\hline 2009 & $\begin{array}{l}\text { New insights into restless genital syndrome: } \\
\text { static mechanical hyperesthesia and neuro- } \\
\text { pathy of the nervus dorsalis clitoridis }\end{array}$ & $\begin{array}{l}\text { Waldinger MD, Venema PL, } \\
\text { Van Gils AP, Schweitzer DH }\end{array}$ & J Sex Med & 23 & $\begin{array}{l}\text { Os autores investigaram, mediante um teste sensório, pontos } \\
\text { de sensibilidade envolvidos no TEGP na região pélvica }\end{array}$ \\
\hline 2009 & $\begin{array}{l}\text { Persistent genital arousal disorder (PGAD): } \\
\text { case report of long-term symptomatic } \\
\text { management with electroconvulsive therapy }\end{array}$ & $\begin{array}{l}\text { Korda JB, Pfaus JG, Kellner } \\
\text { CH, Goldstein I }\end{array}$ & J Sex Med & 1 & $\begin{array}{l}\text { Os autores apresentam um caso de tratamento do TEGP por } \\
\text { meio da eletroconvulsoterapia }\end{array}$ \\
\hline 2009 & $\begin{array}{l}\text { Persistent genital arousal disorder and } \\
\text { trazodone. Morphometric and vascular } \\
\text { modifications of the clitoris. A case report }\end{array}$ & Battaglia C, VenturoliS & J Sex Med & 1 & $\begin{array}{l}\text { Os autores apresentam um caso em que a paciente passou } \\
\text { a exibir os sintomas após o tratamento com trazadona, com } \\
\text { alterações no tamanho do clitóris }\end{array}$ \\
\hline 2009 & $\begin{array}{l}\text { Report of spontaneous and persistent } \\
\text { genital arousal in women attending a sexual } \\
\text { health clinic }\end{array}$ & $\begin{array}{l}\text { Garvey LJ, West C, Latch N, } \\
\text { Leiblum S, Goldmeier D }\end{array}$ & Int J STD AIDS & 96 & $\begin{array}{l}\text { Os autores tentam estabelecer a frequência com que o TEGP } \\
\text { ocorre, numa população atendida numa clínica sexual em } \\
\text { Londres }\end{array}$ \\
\hline 2009 & $\begin{array}{l}\text { Persistent Genital Arousal Disorder: a case } \\
\text { report in a woman with lifelong PGAD } \\
\text { where serendipitous administration of } \\
\text { varenicline tartrate resulted in symptomatic } \\
\text { improvement }\end{array}$ & $\begin{array}{l}\text { Korda JB, Pfaus JG, } \\
\text { Goldstein I }\end{array}$ & J Sex Med & 1 & $\begin{array}{l}\text { Os autores descrevem um caso em que a paciente experimen- } \\
\text { tou remissão dos sintomas do TEGP após o uso da vareniclina } \\
\text { para tratamento de tabagismo }\end{array}$ \\
\hline 2009 & $\begin{array}{l}\text { FSFI scores of women with persistent genital } \\
\text { arousal disorder compared with published } \\
\text { scores of women with female sexual arousal } \\
\text { disorder and healthy controls }\end{array}$ & Leiblum SR, Seehuus M & J Sex Med & 431 & $\begin{array}{l}0 \text { objetivo dos autores foi comparar os escores do FSFI entre } \\
\text { três grupos distintos: mulheres com disfunção sexual, com } \\
\text { TEGP e controles, para identificar se as mulheres com TEGP têm } \\
\text { uma piora na sua função sexual }\end{array}$ \\
\hline 2009 & $\begin{array}{l}\text { Persistent genital arousal disorder in } 18 \\
\text { Dutch women: Part I. MRI, EEG, and transva- } \\
\text { ginal ultrasonography investigations }\end{array}$ & $\begin{array}{l}\text { Waldinger MD, Van Gils AP, } \\
\text { Ottervanger HP, Vanden- } \\
\text { broucke WV, Tavy DL }\end{array}$ & J Sex Med & 18 & $\begin{array}{l}\text { Apresentados resultados de exames realizados em grupo } \\
\text { de mulheres com diagnóstico de TEGP, buscando esclarecer } \\
\text { possíveis causas do transtorno }\end{array}$ \\
\hline 2009 & $\begin{array}{l}\text { Persistent genital arousal disorder in } 18 \\
\text { Dutch women: Part II. A syndrome clustered } \\
\text { with restless legs and overactive bladder }\end{array}$ & $\begin{array}{l}\text { Waldinger MD, Schweitzer } \\
\text { DH }\end{array}$ & J Sex Med & 18 & $\begin{array}{l}\text { Os autores apresentam resultados de exames realizados em } \\
\text { um grupo de mulheres com diagnóstico de TEGP, buscando } \\
\text { esclarecer possíveis causas do transtorno }\end{array}$ \\
\hline 2009 & $\begin{array}{l}\text { Successful transcutaneous electrical nerve } \\
\text { stimulation in two women with restless } \\
\text { genital syndrome: the role of ad- and } \\
\text { c-nerve fibers }\end{array}$ & $\begin{array}{l}\text { Waldinger MD, De Lint GJ, } \\
\text { Venema PL, Van Gils AP, } \\
\text { Schweitzer DH }\end{array}$ & J Sex Med & 2 & $\begin{array}{l}\text { Os autores apresentam dois casos de pacientes com TEGP } \\
\text { tratadas de maneira eficaz mediante estimulação do nervo } \\
\text { pudendo e discutem o papel desse no transtorno }\end{array}$ \\
\hline 2009 & $\begin{array}{l}\text { Restless genital syndrome before and after } \\
\text { clitoridectomy for spontaneous orgasms: a } \\
\text { case report }\end{array}$ & $\begin{array}{l}\text { Waldinger MD,Venema PL, } \\
\text { Van Gils AP, Schutter EMJ, } \\
\text { Schweitzer DH }\end{array}$ & J Sex Med & 1 & $\begin{array}{l}\text { Os autores relatam um caso em que a paciente com TEGP optou } \\
\text { pela cliteridoctomia, não obtendo melhora no quadro clínico. } \\
\text { Os autores descartam o procedimento como tratamento do } \\
\text { transtorno }\end{array}$ \\
\hline 2010 & $\begin{array}{l}\text { Physical therapy treatment of persistent } \\
\text { genital arousal disorder during pregnancy: } \\
\text { a case report }\end{array}$ & Rosenbaum TY & J Sex Med & 1 & $\begin{array}{l}\text { Relato de caso de uma paciente com sintomas de TEGP durante } \\
\text { a gravidez, que, após exercícios físicos, apresentou remissão } \\
\text { dos sintomas }\end{array}$ \\
\hline 2010 & $\begin{array}{l}\text { Persistent genital arousal disorder associa- } \\
\text { ted with functional hyperconnectivity of an } \\
\text { epileptic focus }\end{array}$ & $\begin{array}{l}\text { Anzellotti F, Franciottl R, } \\
\text { Bonanni L, Tamburro G, } \\
\text { Perruci MG, Thomas A, } \\
\text { Pizzela V, Romani GL, } \\
\text { Onofrj M }\end{array}$ & Neuroscience & 1 & $\begin{array}{l}0 \text { artigo descreve um caso de TEGP associado a um foco } \\
\text { epiléptico localizado no giro cingular esquerdo }\end{array}$ \\
\hline
\end{tabular}

\section{Estudos de caso}

Em 2001, Leiblum e Nathan6 relataram pela primeira vez na literatura uma condição com ocorrência observada somente em mulheres, caracterizada por respostas fisiológicas correspondentes à fase de excitação sexual, que permanecia por horas ou mesmo dias, apesar dos esforços para remissão dos sintomas. As mulheres não reconheciam sentimentos subjetivos de desejo e não eram capazes de identificar gatilhos que disparavam as sensações fisiológicas, além de considerarem essas sensações como intrusivas, indesejáveis e espontâneas.

Nesse primeiro artigo, as autoras relataram seis casos de mulheres com a mesma sintomatologia, cujas idades variavam entre 35 e 81 anos. As autoras primeiramente nomearam esse quadro clínico de "persistent sexual arousal syndrome", ou síndrome da excitação sexual persistente.
Em 2003, após o $2^{\text {nd }}$ International Consultation on Sexual Medicine: Sexual Dysfunction in Men and Women, realizado em Paris, um grupo de especialistas propôs que o transtorno integrasse uma nova classificação diagnóstica provisória e definiram o transtorno como "excitação genital espontânea, intrusiva e indesejada na ausência de interesse ou desejo sexual. Qualquer consciência de excitação subjetiva é tipicamente desagradável. A excitação não é aliviada por um ou mais orgasmos e as sensações de excitação persistem por horas ou dias" ${ }^{\prime \prime}$.

Há relatos de associação entre o transtorno e o excesso de consumo de alimentos à base de soja ${ }^{8}$, de exacerbação de sintomas durante o sono em uma mulher na menopausa ${ }^{9}$ e da possível associação entre a retirada de medicamentos antidepressivos inibidores seletivos de recaptação de serotonina (ISRS) e o surgimento de sintomas do transtorno ${ }^{10}$. 
Craig $^{7}$ apresentou uma proposta de cinco subcategorias diagnósticas para o transtorno, baseadas na hipótese etiológica principal, ou seja, o fator causador do TEGP. Seriam elas: 1. hipersensibilidade pélvica/sexual - há um dramático e inapropriado aumento das sensações neurológicas sexuais em uma excitação intensa e prolongada; 2. variante da síndrome de congestão pélvica - há uma dilatação desorganizada no sistema de veias responsáveis por drenar o sangue da pélvis e órgãos genitais, levando a uma congestão sanguínea desses locais; 3. tipos neurológicos de TGEP - tanto por uma falha neurológica causada por uma lesão sutil ou alteração na resposta de um neurotransmissor; 4. TEGP associado ao endócrino - fase refratária ausente ou mínima após o orgasmo ou aumento da excitação sexual decorrente de problemas hormonais; o início pode coincidir com o princípio da menopausa ou os sintomas podem estar presentes em determinadas fases do ciclo menstrual; e 5. TEGP variante da síndrome de Tourette associado com tiques, masturbação compulsiva, pensamentos intrusivos e histórico familiar de Tourette ou transtorno similar. Tecnicamente, seria um tipo de transtorno obsessivocompulsivo (TOC) com sintomas similares ao TEGP.

Em 2006, um grupo de pesquisadores apresentou um estudo de caso em que duas pacientes com diagnóstico de TEGP e de transtorno bipolar foram tratadas com eletroconvulsoterapia (ECT), obtendo-se uma resposta positiva e a diminuição dos sintomas. O trabalho ainda relata que as duas pacientes começaram a experimentar os sintomas enquanto usavam lamotrigina, mas não foi possível correlacionar o uso da medicação com o surgimento dos sintomas ${ }^{11}$.

Em 2009, outro artigo descreveu ${ }^{12}$ um caso em que a ECT obteve um resultado satisfatório no tratamento do TEGP. A paciente, inicialmente, foi tratada de depressão, fazendo uso de diferentes medicamentos antidepressivos (proptilina, maprotilina e bupropiona).

Essa paciente teve início precoce da menopausa (aos 42 anos), em que desenvolveu um quadro de hipotireoidismo, uma vez que fez reposição hormonal. Por continuar apresentando sintomas depressivos, a paciente foi medicada com paroxetina, que, apesar de melhorar os sintomas depressivos, afetou sua capacidade orgástica. Nesse momento, ela foi diagnosticada com transtorno bipolar tipo II e sua medicação foi abruptamente modificada para lamotrigina. Com a interrupção da paroxetina, ela começou a experimentar excitação genital indesejada, não relacionada a quaisquer estímulos ou pensamentos sexuais.

Por não obter alívio nos seus sintomas, a paciente foi indicada para tratamento com ECT, em que se obteve uma melhora em seu quadro clínico. Foi submetida a 30 sessões de ECT durante quatro anos, mas em sua última sessão ela experimentou o que chamou de "viagem ruim" e uma perda do senso pessoal e espacial. A hipótese levantada no artigo é que, após a retirada abrupta da paroxetina, um ISRS, houve uma deficiência temporária nas sinapses do 5-HT. Essa mes- ma paciente recebeu, aos 30 anos, o diagnóstico da síndrome das pernas inquietas (SPI).

Um outro artigo ${ }^{13}$ também apresentou um relato de caso no qual uma paciente tratada de transtorno bipolar, após não obter resposta ao tratamento com lítio e quetiapina, iniciou tratamento com venlafaxina, aumentada gradualmente. Quando a dosagem da medicação alcançou 300 mg, a paciente passou a perceber um aumento de sensações no clitóris dissociadas de qualquer estímulo ou desejo sexual. A paciente não se encontrava em estado maníaco ou hipomaníaco e seu humor era depressivo. Com a diminuição da medicação, os sintomas desapareceram.

Bell et al..$^{14}$ relataram um caso de paciente com defeito septal atrial, tratada com fludrocortisona para hipotensão e bradicardia. Nesse artigo, é sugerido que a combinação da doença cardíaca com o tratamento com fludrocortisona possa ter aumentado os níveis do peptídeo natriurético atrial, o qual possui um efeito vasodilatador, contribuindo para o surgimento e a manutenção do transtorno.

Ao se reavaliarem os resultados de duas pesquisas conduzidas na internet, Leiblum e Goldmeier publicaram um artigo relatando cinco casos de mulheres que indicavam o início de seus sintomas com a retirada de medicamentos antidepressivos ISRS ${ }^{15}$.

Dos cinco casos relatados, quatro fizeram uso de venlafaxina, que já havia sido citada na literatura como fator propiciador para o desenvolvimento do transtorno. A literatura aponta que medicamentos antidepressivos podem causar sintomas colaterais quando descontinuados, e nesse artigo os autores sugerem que a retirada da medicação ISRS possa causar um aumento da liberação do peptídeo natriurético atrial, o que resulta em um aumento da vasodilatação da vulva, porém não explicam os motivos pelos quais essa vasodilatação se restringe à região genital.

Mais uma série de seis casos de TEGP foi relatada por Goldmeier e Leiblum ${ }^{16}$. As idades das mulheres variavam entre 29 e 62 anos, e os casos não possuíam características similares no histórico clínico nem no ginecológico, psicológico ou relativo ao início dos sintomas. Apenas duas pacientes relataram sintomas obsessivo-compulsivos e ansiosos e outra tinha um quadro de ansiedade grave. Os autores propõem que um ingurgitamento vaginal, aliado às patologias dermatológicas, em conjunto com quadros ansiosos, pode favorecer o surgimento dos sintomas do TEGP.

Foi relatado, ainda, o caso de uma mulher de 46 anos, bissexual, nulípara, com histórico de TEGP durante toda a vida (de acordo com seu autorrelato, os sintomas estavam presentes desde os 12 anos, com notável aumento nos grandes lábios). Obteve-se melhora significativa do transtorno quando foi usada vareniclina, um medicamento utilizado no combate ao tabagismo que causa diminuição nos níveis extracelulares de dopamina. Após duas semanas de tratamento com a medicação e com a suspensão do uso do cigarro, os 
sintomas diminuíram consideravelmente e, com dois meses de uso, os sintomas desapareceram ${ }^{17}$.

Após a suspensão da medicação por parte da paciente, os sintomas retornaram. As hipóteses levantadas nesse estudo são duas: 1. o TEGP seria resultado de uma hiperestimulação na liberação central de dopamina; 2 . a restauração de um funcionamento normal foi resultado da ação da vareniclina diminuindo a liberação da dopamina.

Battaglia e Venturoli ${ }^{18}$ relatam o caso de uma mulher, de 29 anos, sem histórico de problemas ginecológicos, abuso sexual, uso de cigarro ou álcool, com episódio depressivo/ ansioso ocorrido dois anos antes, durante o qual fez uso de trazodona. A paciente percebeu que, depois de um mês usando o medicamento, seu impulso sexual havia aumentado e, logo após, ela começou a experimentar excitação genital espontânea e indesejada, que não era aliviada por um ou mais orgasmos. Os orgasmos eram provocados por qualquer leve fricção dos genitais. A medicação foi suspensa por conta dos sintomas e não houve substituição por nenhum outro medicamento para tratar a depressão e a ansiedade.

Apesar da diminuição da frequência dos orgasmos espontâneos e da excitação genital indesejada, a paciente continuava experimentando-os, dois anos depois do fim do tratamento. A trazodona possui alto efeito vasodilatador $\mathrm{e}$ está associada a casos de priapismo. Os autores sugerem que casos de priapismo clitorial possam favorecer o sentimento de persistência da excitação genital, bem como orgasmos espontâneos, indesejados.

Mais recentemente, um artigo apresentou um caso de TEGP associado a um foco epiléptico localizado no giro cingular esquerdo ${ }^{19}$. Em outro artigo, foi descrita a intervenção por meio de fisioterapia no nervo pudendo em uma paciente de 27 anos, grávida, exibindo sintomas de TEGP. Após uma semana de tratamento, a paciente experimentou a remissão completa dos sintomas ${ }^{20}$. Outro artigo apresentou dois casos em que a estimulação elétrica transcutânea do nervo obteve resultados satisfatórios no tratamento do transtorno ${ }^{21}$.

Waldinger et al. ${ }^{22}$ apresentaram um caso no qual uma paciente de 77 anos, mesmo após uma clitoridectomia, continuava apresentando sensações genitais condizentes com o transtorno. A paciente foi examinada por meio de EEG, ressonância magnética cerebral e genital, e do teste de mapeamento tátil genital. A ressonância genital mostrou varizes pélvicas e uma cicatriz no clitóris. Os testes sensoriais acusaram diversos pontos de hiperestesia mecânica estática, do lado esquerdo do nervo pudendo.

Os autores concluíram que o quadro da paciente era decorrente de uma neuropatia do nervo pudendo e que, apesar de algum alívio nos orgasmos espontâneos, a clitoridectomia não era eficaz para a eliminação dos sintomas associados ao TEGP.

Em 2008, foi publicado um artigo que relacionava a síndrome da congestão pélvica, um quadro caracterizado por maior congestão pélvica resultante do aumento no volume das veias presentes nessa região, com o TEGP23. Uma mulher de 62 anos, com quatro gravidezes e três partos, apresentava sensação persistente de excitação sexual, desconectada de qualquer sentimento ou desejo sexual, intrusiva e indesejada, e exacerbada por ficar em pé, sentar, caminhar e com alívio parcial ao deitar.

O orgasmo e o ato de urinar promoviam alívio dos sintomas também. $O$ exame de ressonância magnética demonstrou dilatação da veia ovariana esquerda e presença de varizes na pélvis e parede vaginal. Até então, esses dois quadros não haviam sido relacionados na literatura.

Um grupo de pesquisadores conduziu um estudo longitudinal com 18 mulheres atendidas no Ambulatório de Neurosexologia do HagaHospital Leyenburg, Holanda, entre outubro de 2004 e julho de 2008, todas previamente diagnosticadas com TEGP. Os resultados do estudo foram divulgados em duas partes. No primeiro artigo ${ }^{24}$, os autores apresentam os resultados dos exames de ressonância magnética do cérebro e da pélvis, eletroencefalograma e ultrassonografia transvaginal, caso a ressonância acusasse anormalidades pélvicas.

Os EEG das mulheres não mostraram anormalidades graves, mas uma paciente exibiu atividade epiléptica. As ressonâncias magnéticas cerebrais não apresentaram anormalidades. As pélvicas não indicaram alterações no clitóris ou na uretra. Das 18 mulheres do estudo, 10 apresentaram varizes pélvicas; em 4, elas foram consideradas varizes graves e 3 demonstraram dilatações como percebidas na síndrome da congestão pélvica.

Além de varizes pélvicas, 7 mulheres possuíam varizes em uma ou duas pernas. Comparando a taxa de prevalência de varizes pélvicas em mulheres sem diagnóstico de TEGP, a taxa de 55\% encontrada nesse estudo é relativamente alta, sugerindo que as varizes pélvicas tenham papel importante na etiologia do transtorno.

No segundo artigo com os resultados da pesquisa, os autores apresentam outras características da população estudada ${ }^{25}$. As mulheres foram acompanhadas, recebendo psicoterapia breve nos momentos de crise. A terapia cognitivo-comportamental foi descartada, porque, segundo os autores, essas mulheres experimentavam muita confusão, impedindo-as de seguir corretamente as instruções, como é característico dessa linha de terapia.

A maioria das pacientes não relatou histórico de doenças mentais, mas houve relatos de episódio depressivo único, síndrome de burnout, anorexia nervosa, abuso etílico e transtorno de ajustamento. Três das mulheres acompanhadas foram abusadas sexualmente quando mais jovens, mas não relacionam seus problemas a esse histórico.

Dezesseis mulheres nunca haviam feito uso de medicação antidepressiva, e cinco começaram a fazer uso após o surgimento dos sintomas, para tratamento da depressão associada ao TEGP. Entre os gatilhos citados no estudo estão: 
tensão (71\%), se sentir extremamente amedrontada (36\%), raiva (21\%), ansiedade aguda (14\%), aborrecimento (14\%) e estresse agudo (14\%). Duas mulheres relataram que o TEGP podia ser disparado pelo consumo de álcool, especialmente de vinho tinto.

Os autores investigaram a presença da SPI e também da síndrome da bexiga hiperativa ${ }^{25}$ e encontraram que 12 das 18 mulheres experimentaram sintomas de pernas e até de braços inquietos. Foi avaliada a taxa de ferro no organismo dessas mulheres, visto que nas pacientes diagnosticadas com essa síndrome a taxa de ferro é elevada, porém nada foi encontrado.

O estudo demonstrou uma prevalência muito alta de "pernas inquietas" (67\%), sintomas de bexiga hiperativa (67\%), varizes pélvicas (55\%) e varizes de membros inferiores (39\%), superior a estudos com mulheres que não apresentam o diagnóstico de TEGP, e os autores propuseram a seguinte hipótese: O TEGP seria um quadro clínico, complexo, de distesias e parestesias equivalentes às sensações nos membros inferiores na SPI. Juntamente com a síndrome da bexiga hiperativa, esses quadros seriam expressão de uma síndrome que os autores propõem chamar de síndrome dos genitais inquietos (SGI). Os autores listam uma série de 16 motivos pelos quais essas síndromes deveriam ser agrupadas em um quadro clínico mais complexo. Eles também apresentam dados que sugerem uma eficácia do clonazepam no tratamento do transtorno.

Em mais um artigo do grupo de pesquisa de Waldinger et al. ${ }^{26}, 23$ mulheres foram avaliadas em busca da identificação das regiões genitais envolvidas no transtorno. A região genital foi sistematicamente investigada por meio do toque de um cotonete, buscando respostas táteis. Cada ponto de pressão foi avaliado e pediu-se às pacientes que relatassem as sensações evocadas. Esse teste foi chamado de teste de mapeamento tátil genital.

As pacientes foram encaminhadas à pesquisa por seus médicos e, para o diagnóstico de TEGP, todos os cinco critérios diagnósticos já estabelecidos na literatura deveriam estar presentes. Doze mulheres fizeram parte do primeiro estudo do grupo. Quinze das mulheres estavam na menopausa. A média de idade do início dos sintomas foi de 50 anos. Dessas mulheres, dezoito relataram ser portadoras da SPI, uma relatou braços inquietos; oito possuíam os sintomas há muitos anos. Dez mulheres relataram que os sintomas de SPI começaram após os sintomas de TEGP.

Entre essas mulheres também foi encontrada alta prevalência de síndrome da bexiga hiperativa (16 mulheres); sete mulheres relataram que o início dos sintomas ocorreu após o surgimento dos sintomas do TEGP. O teste de mapeamento tátil genital resultou em diferentes pontos de disparo do orgasmo em cada mulher e mostrou sensibilidade mecânica estática ao redor dos genitais, dos lábios, clitóris e acima do osso púbico.
Os autores questionam o papel do nervo dorsal do clitóris no SGl, bem como o envolvimento do nervo pudendo ${ }^{26}$, já que muitas mulheres relataram sensações genitais ao toque do dedo na região onde o nervo está localizado. Aparentemente, ele evoca sensações pré-orgásticas e orgásticas nas mulheres, mesmo sem presença de desejo sexual ou fantasias.

\section{Estudos epidemiológicos}

Em uma tentativa de esclarecer o transtorno, suas características, comorbidades e epidemiologia, Leiblum et al. ${ }^{27}$ conduziram uma pesquisa, pela internet, utilizando em questionário desenvolvido especialmente para o estudo. Esse questionário estava disponível em três websites sobre saúde feminina, acessível a todos os visitantes, que, após lerem algumas informações sobre o transtorno, podiam respondê-lo anonimamente.

Cento e sete pessoas responderam à pesquisa, mas somente 103 questionários foram considerados para as análises estatísticas. Dentre os dados encontrados, destaca-se que 53\% dos respondentes satisfaziam os cinco critérios do transtorno. Nesses sujeitos, os sintomas mais comuns foram: congestão $(74,8 \%)$, formigamento $(78,6 \%)$, umidade $(75,7 \%)$, latejamento $(72,2 \%)$ e contrações $(35,4 \%)$.

A maioria das respondentes havia engravidado pelo menos uma vez. Os dados encontrados também sugerem que esse grupo de mulheres sofre mais de humor deprimido $(42,7 \%)$, ataque de pânico e ansiedade $(31,1 \%)$, dores de cabeça $(23,3 \%)$, comportamentos e pensamentos obsessivos (22,3\%) e hipertensão $(13,6 \%)$.

Um novo estudo epidemiológico foi conduzido para tentar identificar os correlatos médicos, psicológicos e farmacológicos envolvidos no TEGP28. Por meio de um questionário na internet, foram identificados dois grupos de mulheres: grupo TEGP e grupo não TEGP. No grupo TEGP, as mulheres endossavam todos os cinco critérios estabelecidos para o transtorno, enquanto no outro grupo as mulheres endossavam apenas alguns desses critérios.

Existem limitações nesse tipo de estudo, visto que não é possível confirmar os dados obtidos. As mulheres forneciam dados sobre gravidez, histórico sexual e ginecológico, abuso sexual, injúria genital, dados sociodemográficos e descrições sobre seus sintomas e sentimentos em relação ao transtorno.

Os dados foram colhidos durante um ano e os resultados obtidos demonstraram que as mulheres que apresentavam todos os critérios do transtorno estavam mais propensas à depressão, a transtornos ansiosos e a sintomas obsessivocompulsivos, como monitoramento excessivo de alterações fisiológicas corporais. O estudo não encontrou evidências de associação entre qualquer condição médica ou psicofármacos com o relato de TEGP28.

Um estudo desenvolvido em uma clínica de saúde sexual na Inglaterra buscava identificar a frequência e a natureza do 
TEGP numa população feminina com idade superior a 18 anos $^{29}$. Para conduzir o estudo, os pesquisadores utilizaram uma versão adaptada do questionário on-line usado por Leiblum em suas pesquisas via internet ${ }^{28}$.

Cento e cinquenta e três mulheres foram convidadas a participar desse estudo e 96 concordaram em participar, dando consentimento por escrito ${ }^{29}$. A idade das mulheres variava entre 18 e 64 anos, com média de 28 anos. Trinta e três por cento das mulheres que responderam à pesquisa relataram pelo menos um sinal de excitação genital excessiva. Do total também, 19 mulheres relataram ter experimentado sensações de excitação genital não relacionada à excitação ou ao desejo sexual.

Apenas uma mulher de 38 anos preencheu os cinco critérios do transtorno. Ela tinha um histórico de depressão, ansiedade, ataques de pânico, síndrome do intestino irritável e anemia, além de já ter sido violentada sexualmente. Os sintomas relatados foram inchaço dos genitais, ereção do mamilo e aflição, estando presentes há anos.

Os autores pontuam que é interessante que um número significativo de mulheres relate excitação genital sem a consciência de desejo ou estímulos sexuais, quando de acordo com o modelo do ciclo de resposta sexual adotado, em que a excitação/desejo sexual tem um papel central29.

Essa ocorrência de excitação genital espontânea pode sugerir que essa característica é parte da resposta normal da sexualidade feminina. Os autores discutem que o número de sujeitos da pesquisa não permite generalizações a respeito dos achados.

Numa tentativa de comparar o TEGP com TESF, foi feita uma análise dos escores do Female Sexual Function Index (FSFI) ${ }^{30}$ de três grupos: pacientes com TEGP, TESF e um grupo de controle sem diagnóstico ${ }^{31}$. Para isso, foram utilizados os questionários de TEGP e os dados obtidos na validação do FSFI.

Não havia dados dos grupos de controle e TESF sobre a prevalência de transtornos depressivos ou ansiosos disponíveis para comparação. Em todas as subescalas, as mulheres com TGEP ficaram entre o grupo de controle e o grupo com TESF e não obtiveram escore mínimo para diagnóstico de disfunção sexual, sugerindo que essas mulheres não são sexualmente disfuncionais, mas também não apresentam níveis de satisfação como as mulheres do grupo de controle ${ }^{31}$.

\section{Mudança na nomenclatura}

Inicialmente, a condição foi considerada um problema de ordem sexual, do qual se originava sua nomenclatura, persistent sexual arousal syndrome, ou síndrome da excitação sexual persistente. Porém, após novos relatos de caso e de um maior conhecimento a respeito do problema, foi observado que ele era relacionado mais à excitação genital do que sexual.

As mulheres descreviam o transtorno como preocupante e aflitivo, em vez de sexualmente satisfatório. Todas as iniciativas sexuais se davam não em busca do prazer, mas, sim, de alívio dos sintomas genitais, que eram extremamente perturbadores. Com isso, Leiblum passou a considerar o problema como de ordem genital mais do que sexual, renomeando a condição para "persistent genital arousal disorder", ou transtorno da excitação genital persistente (TEGP) ${ }^{32}$.

Baseado em seus achados, Waldinger propôs que o TEGP seja incluído em um espectro de doenças juntamente com a SPI e a síndrome da bexiga hiperativa ${ }^{25}$. Para tanto, propôs a mudança de nomenclatura desse espectro para síndrome dos genitais inquietos (SGI). Para este artigo, consideraremos a nomenclatura proposta por Leiblum, já que esta se refere somente ao transtorno específico, e não a possíveis associações a outros quadros clínicos.

\section{Mecanismos etiopatogênicos}

Apesar dos avanços no conhecimento do transtorno, não há consenso na literatura sobre as possíveis causas e fatores de risco envolvidos no surgimento e manutenção destes. Algumas hipóteses foram listadas na literatura, baseadas nos relatos de casos disponíveis ${ }^{28}: 1$. alterações neurológicas centrais (exemplo: pós-injúria, lesões cerebrais específicas); 2. alterações neurológicas periféricas (hipersensibilidade do nervo pélvico); 3. alterações vasculares (congestão pélvica, varizes pélvicas); 4. pressão mecânica sobre estruturas genitais; 5. alterações induzidas por medicação; 6. alterações psicológicas (estresse, ansiedade) ou, ainda, uma combinação de todos ou alguns fatores.

Leiblum descreveu dois subtipos do transtorno ${ }^{32}$ : um grupo de mulheres que interpreta esses sintomas como parte de sua natureza sexual, sem avaliações negativas a respeito deles. Ela chamou esse grupo de Não TEGP, ou seja, as mulheres, apesar de relatarem os sintomas característicos, não os avaliavam como aflitivos ou intrusivos.

Já o outro grupo, chamado de grupo TEGP, interpreta esses sintomas de maneira negativa, associando-os com estados afetivos negativos. Essas mulheres seriam mais propensas a transtornos depressivos e ansiosos, além de exibirem sintomas obsessivo-compulsivos.

Baseadas nesse achado, Leiblum e Chivers $^{33}$ propuseram que para algumas mulheres os incômodos psicológicos decorrentes do transtorno seriam mais desconfortáveis do que os fisiológicos ${ }^{33}$. Para isso, a ansiedade teria um papel fundamental na manutenção do TEGP.

É conhecido que a ansiedade aumenta a atividade do sistema nervoso autonômico e o estreitamento cognitivo, levando a hiperatividade fisiológica e exacerbação de estados emocionais negativos como medo e ansiedade. Esse mecanismo poderia contribuir para manter e intensificar os sintomas fisiológicos.

Após experimentarem os sintomas pela primeira vez e avaliá-los negativamente (baseadas em seus próprios sistemas de crenças a respeito de si e do mundo), essas mulheres se tornariam hipervigilantes a quaisquer possíveis alterações 
físicas na região genital. O modelo proposto envolveria ${ }^{33}$ : 1. detecção de uma resposta genital espontânea; 2 . avaliação dessa resposta como inapropriada ou negativa; 3. experiência da ansiedade relacionada à avaliação; 4 . a ansiedade, então, aumentaria a atividade do sistema nervoso simpático, o que aumentaria a percepção das respostas genitais, consequentemente sendo focada ainda mais atenção às sensações genitais. As autoras ainda relacionam esse modelo ao modelo cognitivo do TOC.

\section{DISCUSSÃO}

A literatura disponível sobre o transtorno baseia-se principalmente em relatos de caso. Os relatos oferecem a oportunidade de levantar hipóteses em relação aos fatores psicológicos, neurológicos, fisiológicos, farmacológicos e vasculares envolvidos na etiologia do transtorno, porém, como muitos dos casos apresentam etiologia diversa, não foi possível chegar a um consenso sobre que fatores estão envolvidos no surgimento e na manutenção do TEGP.

Os casos apontam para uma relação entre o transtorno e o uso de medicação antidepressiva, mas não é possível estabelecer uma relação temporal entre os dois. Alguns estudos $^{12,15}$ relatam o surgimento após a retirada da medicação, enquanto outros ${ }^{11,18}$ relatam a coincidência entre o início do uso da medicação e o surgimento dos sintomas. Nem todos os casos relatam uso de medicação antidepressiva. Sugere-se que outros fatores possam estar associados ao transtorno.

Os estudos de Waldinger et al. ${ }^{24} \mathrm{e}$ Waldinger e Schweit$z^{2} r^{25}$ sugerem que alterações físicas como varizes na região pélvica tenham papel fundamental no surgimento do transtorno e que a presença de varizes seja um fator de risco do TEGP. A prevalência de varizes entre as mulheres estudadas por Waldinger é superior à encontrada na literatura, além da comorbidade entre o TEGP e a SPI e a síndrome da bexiga hiperativa.

É importante frisar que, para o diagnóstico do transtorno, é necessária a presença de pelo menos um grau moderado de angústia. Leiblum et al.. ${ }^{77,32}$ e Leiblum e Chivers ${ }^{33}$, em seus estudos, perceberam que muitas mulheres relatam respostas fisiológicas de excitação espontânea, mas nem todas as interpretam negativamente. Isso evidencia que a ansiedade possui um papel determinante na manutenção do quadro, aumentando ainda mais a consciência dessas mulheres em relação a pequenas alterações fisiológicas em sua região genital.

\section{CONCLUSÃO}

Apesar do esforço em prol de um maior entendimento do transtorno da excitação genital persistente, pouco foi elu- cidado sobre etiologia, fatores de risco e epidemiologia do problema. A literatura disponível sobre o tema baseia-se, principalmente, em estudos de casos isolados que apresentam algumas características em comum, mas não esclarecem os fatores de risco para o desenvolvimento e manutenção do transtorno.

Os poucos estudos com características epidemiológicas ou foram desenvolvidos via internet, o que pode limitar o estudo, ou ainda com um pequeno número de pacientes, o que, apesar de propiciar o desenvolvimento de novas hipóteses, não é suficiente para corroborá-las nem permite generalização dos achados.

Ainda existe um grande desconhecimento do transtorno por parte dos profissionais da área da saúde e é muito provável que diversos casos sejam diagnosticados erroneamente ou ainda ignorados, dificultando ainda mais uma melhor compreensão do problema.

\section{REFERÊNCIAS}

1. American Psychiatry Association. Manual Diagnóstico e Estatístico de Transtornos Mentais. 4. ed. rev. Porto Alegre: Artmed; 2002.

2. Organização Mundial da Saúde. Classificação de Transtornos Mentais e de Comportamento da CID-10: descrições clínicas e diretrizes diagnósticas. Porto Alegre: Artes Médicas; 1993.

3. Masters WM, Johnson VE. A conduta sexual humana. 3. ed. Rio de Janeiro: Civilização Brasileira; 1979 .

4. Kaplan HS. A nova terapia do sexo. v. 2. Rio de Janeiro: Nova Fronteira; 1983.

5. Basson R, Leilblum S, Brotto L, Derogatis L, Fourcroy J, Fulgl-Meyer K, et al. Revised definitions of women's sexual dysfunction. J Sex Med. 2004;1:40-8.

6. Leiblum SR, Nathan SG. Persistent sexual arousal syndrome: a newly discovered pattern of female sexuality. J Sex Marital Ther. 2001;27:365-80.

7. Craig HR. Persistent sexual arousal syndrome (PSAS): possible etiologies and potential therapies. 2006. Disponível em: http://f1.grp.yahoofs.com/v1/YE_HTDbjsPKTZMDB1UZWpkCfvWidDDTNR-ZS0qF6q1K1ByOMCUPuXgvIQHwwV2FuN4N3b88zAb0_92jwkCZyaNb9qoLgqQa3Q/Hormone/PSAS_and_Hormones_Dr.-Randall\%3DCraig.pdf

8. Amsterdam A, Abu-Rustum N, Carter J, Krychman M. Persistent sexual arousal syndrome associated with increased soy intake. J Sex Med. 2005;2:338-40.

9. Wylie K, Levin R, Hallam-Jones R, Goddard A. Sleep exacerbation of persistent sexual arousal syndrome in a postmenopausal woman. J Sex Med. 2006;3:296-302.

10. Goldmeier D, Bell C, Richardson D. Withdrawal of selective serotonin reuptake inhibitors (SSRIS) may cause increased atrial natriuretic peptide (ANP) and persistent sexual arousal in women? J Sex Med. 2006;3:376

11. Yero SA, McKinney T, Petrides G, Goldstein I, Kellner CH. Successful Use of electroconvulsive therapy in 2 cases of persistent sexual arousal syndrome and bipolar disorder. J ECT. 2006;22:274-5.

12. Korda JB, Pfaus JG, Keller CH, Goldstein I. Persistent genital arousal disorder (PGAD): case report of long-term symptomatic management with electroconvulsive therapy. J Sex Med. 2009;6:2901-9.

13. Mahoney S, Zarate Jr C. Persistent sexual arousal syndrome: a case report and review of the literature. J Sex Marital Ther. 2007;33:65-71.

14. Bell C, Richardson D, Goldmeier D, Crowley T, Kocsis A, Hill S. Persistent sexual arousal in a woman with associated cardiac defects and raised atrial natriuretic peptide. Int J STD AIDS. 2007:18:130-1.

15. Leiblum S, Goldmeier D. Persistent genital arousal disorder in women: case reports of association with anti-depressant usage and withdrawal. J Sex Marital Ther. 2008;34:150-9. 
16. Goldmeier D, Leiblum S. Interaction of organic and psychological factors in persistent genital arousal disorder in women: a report of six cases. Int J STD AIDS. 2008;19:488-90.

17. Korda JB, Pfaus JG, Goldstein I. Persistent Genital Arousal Disorder: a case report in a woman with lifelong PGAD where serendipitous administration of varenicline tartrate resulted in symptomatic improvement. J Sex Med. 2009;6:1479-86.

18. Battaglia C, Venturoli S. Persistent genital arousal disorder and trazodone. Morphometric and vascular modifications of the clitoris. A case report. J Sex Med. 2009;6:2896-900.

19. Anzellotti F, Franciottl R, Bonanni L, Tamburro G, Perruci MG, Thomas A, et al. Persistent genital arousal disorder associated with functional hyperconnectivity of an epileptic focus. Neuroscience. 2010;167:88-96.

20. Rosenbaum TY. Physical therapy treatment of persistent genital arousal disorder during pregnancy: a case report. J Sex Med. 2010:7:1306-10.

21. Waldinger MD, De Lint GJ, Venema PL, Van Gils AP, Schweitzer DH. Successful transcutaneous electrical nerve stimulation in two women with restless genital syndrome: the role of ad- and c-nerve fibers. J Sex Med. 2009;3;1190-9.

22. Waldinger MD, Venema PL, Van Gils AP, Schutter EMJ, Schweitzer DH. Restless genital syndrome before and after clitoridectomy for spontaneous orgasms: a case report. I Sex Med. 2009; 7:1029-34.

23. Thorne C, Stuckey B. Pelvic congestion syndrome presenting as persistent genital arousal: a case report. J Sex Med. 2008;5:504-8.

24. Waldinger MD, Van Gils AP, Ottervanger P, Vandenbroucke WV, Tavy DL. Persistent genital arousal disorder in 18 Dutch women: part I. MRI, EEG, and Transvaginal Ultrasonography Investigations. J Sex Med. 2009,6:474-81.
25. Waldinger MD, Schweitzer DH. Persistent genital arousal disorder in 18 Dutch women: Part II. A syndrome clustered with restless legs and overactive bladder. J Sex Med. 2009;6:482-97.

26. Waldinger MD, Venema PL, Van Gils AP, Schweitzer DH. New insights into restless genital syndrome: static mechanical hyperesthesia and neuropathy of the nervus dorsalis clitoridis. J Sex Med. 2009;6:2778-87.

27. Leiblum S, Brown C, Wan J, Rawlinson L. Persistent sexual arousal syndrome: a descriptive study. J Sex Med. 2005;2:331-7.

28. Leiblum S, Seehuus M, Goldmeier D, Brown C. Psychological, medical, and pharmacological correlates of persistent genital arousal disorder. J Sex Med. 2007;4:1358-66.

29. Garvey LJ, West C, Latch N, Leiblum S, Goldmeier D. Report of spontaneous and persistent genital arousal in women attending a sexual health clinic. Int I STD AIDS. 2009;20: 519-21.

30. Rosen R, Brown C, Heiman J, Leiblum S, Meston C, Shabsigh R, et al. The Female Sexual Function Index (FSFI): a multidimensional self-report instrument for the assessment of female sexual function J Sex Marital Ther. 2000;26:191-208.

31. Leiblum SR, Seehuus M. FSFI scores of women with persistent genital arousal disorder compared with published scores of women with female sexual arousal disorder and healthy controls. J Sex Med. 2009;6:469-73.

32. Leiblum S, Seehus M, Brown C. Persistent genital arousal: disordered or normative aspect of female sexual response? J Sex Med. 2007:4:680-9.

33. Leiblum S, Chivers ML. Normal and persistent genital arousal in women: new perspectives. J Sex Mar Ther. 2007;33;357-73. 